\title{
Counterpoint: Prioritizing Healthcare Workers for Scarce Critical Care Resources Is Impractical and Unjust
}

\author{
Armand Antommaria, MD, PhD, ${ }^{1,2}$ Ndidi I Unaka, MD, MEd ${ }^{1,2 *}$
}

${ }^{1}$ Division of Hospital Medicine, Cincinnati Children's Hospital Medical Center, Cincinnati, Ohio; ${ }^{2}$ Department of Pediatrics, University of Cincinnati College of Medicine, Cincinnati, Ohio.

ran

he impact of the coronavirus disease 2019 (COVID-19) pandemic has been far reaching and devastating. As the pandemic reaches its 1-year mark, there have been more cases and deaths than most of us can comprehend: nearly 28 million cases and 497,000 deaths in the United States ${ }^{1}$ and more than 111 million cases and 2.4 million deaths globally. ${ }^{2}$ Frontline healthcare workers (HCWs) have struggled to provide compassionate care in the face of heavy workloads and risks to themselves and their loved ones. Sadly, more than 1,700 US HCWs have died from COVID-19. ${ }^{3}$ The pandemic has also taken a heavy emotional and psychological toll: HCWs have died by suicide, and others are leaving the profession in which they invested so much and formerly loved. Caring for ill colleagues and dying patients whose family members cannot visit has been particularly difficult. It is, therefore, understandable that some HCWs have called for their prioritization if it becomes necessary to implement crisis standards of care. Although Daffner's ${ }^{4}$ reciprocity argument-HCWs should receive priority because of the risks that they have voluntarily accepted-has some appeal, it disregards several important considerations. First, it fails to consider the changing dynamics of viral transmission during the pandemic or alternative ways in which the duty of reciprocity may be fulfilled that do not involve prioritizing HCWs over others. Second, this position is both over- and underinclusive in ways that make it difficult to implement. Third, and most important, the inordinate attention to the prioritization of HCWs ignores the issues the pandemic raises regarding racism and inequity.

\section{LIMITS OF RECIPROCITY AND ALTERNATIVES TO PRIORITIZATION}

Although the reciprocity argument has some conceptual merit, there are several different ways that the duty of reciprocity can be fulfilled. One fundamental obligation of government agencies and healthcare systems is providing a safe work environment, including adequate personal protective equipment (PPE) and physical distancing. Before we understood the extent of the pandemic, modes of transmission, and effective

*Corresponding Author: Ndidi I Unaka, MD, MEd; Email: Ndidi.Unaka@cchmc.org; Telephone: 513-636-8354; Twitter@NdidiUnaka.

Published online first February 17, 2021.

Received: November 24, 2020; Revised: January 6, 2021;

Accepted: January 21, 2021

(c) 2021 Society of Hospital Medicine DOI 10.12788/jhm.3597 preventative measures, hospital transmission was significant. For example, a single-center case series at Zhongan Hospital of Wuhan University, China, from January 1, 2020, to January 28,2020 , found that $29 \%$ (40 of 138) of hospitalized patients with COVID-19 were health professionals who were presumed to have been infected by patients. ${ }^{5}$ There were also significant shortages of PPE, and a number of frontline HCWs reported being dismissed for calling attention to unsafe conditions. Although professionals have an obligation to expose themselves to risk, they are not obligated to expose themselves to inordinate risk. Prioritizing $\mathrm{HCW}$ in ventilator triage may have been justified during the initial surge.

The use of surgical masks by all employees and patients has substantially reduced hospital transmission. A study at Duke Health, Raleigh, North Carolina, of HCWs who tested positive for SARS-CoV-2 between March 15, 2020, and June 6, 2020, found $22 \%$ of cases were healthcare acquired, $38 \%$ were community acquired, and $40 \%$ were of unknown acquisition route. Of the healthcare-acquired cases, $30 \%$ were thought to be secondary to direct patient care and $70 \%$ to exposure to another worker. The cumulative incidence rate of healthcare-acquired infections among workers decreased significantly 1 week after universal masking was implemented on March 31, 2020. The cumulative incidence rates of community-acquired cases and those with unknown acquisition routes continued to mirror incidence rates in the community. ${ }^{6}$ There is substantially less justification for prioritizing HCWs during the current phase of the pandemic; reciprocity does not justify granting $\mathrm{HCW}$ s infected via community spread greater priority than non-HCWs similarly infected.

There are other means of reciprocating that do not involve prioritization. COVID-19 has exacted an immense toll on the mental well-being of frontline HCWs. They should be provided robust, comprehensive, and accessible mental health services. Additionally, reciprocity can be expressed by providing alternative housing options for HCWs who are concerned about infecting their family members, especially family members at higher risk of morbidity or mortality from COVID-19. Many HCWs have also died from COVID-193; providing life insurance would recognize the sacrifice of $\mathrm{HCW}$ s and support their survivors. None of these interventions would require prioritizing $\mathrm{HCW}$ s over others.

\section{OVER- AND UNDERINCLUSIVENESS}

As Daffner ${ }^{4}$ acknowledges, the category of "healthcare provid$\mathrm{er}^{\prime \prime}$ is both over- and underinclusive. Healthcare providers are exposed to variable risks. Some physicians, for example, are 
no longer involved in direct patient care. It is unclear how triage teams will identify frontline $\mathrm{HCW}$ s or validate claims to being a frontline $\mathrm{HCW}$, especially for individuals not employed by the hospital at which they are seeking care. Hence, triage protocols prioritizing healthcare providers are likely to be substantially overinclusive, which raises significant issues of fairness.

Moreover, the category "healthcare provider" is also underinclusive. Many essential, nonclinical hospital employees expose themselves to risk, including custodial and food service staff. As Daffner ${ }^{4}$ recognizes, there are also many other occupations outside of healthcare in which individuals voluntarily expose themselves to risks for the benefit of others, including police officers, firefighters, and clerks in grocery stores. We would add that workers in the food-supply system, transportation, and education face similar risks. ${ }^{7}$ Identifying the types of jobs that should confer priority and validating an individual's employment also makes implementation difficult and risks injustice.

\section{EQUITY AND JUSTICE}

The COVID-19 pandemic and the murder of Black people by police have brought substantial attention to racism and racial inequities in the United States. We must, however, move from merely acknowledging existing inequities to dismantling structures that perpetuate them. The prioritization of HCWs may further privilege those who already have substantial advantages. This is especially true for physicians. For example, although state and federal laws pose limitations, physicians have historically extended one another professional courtesy by providing free or discounted services. Furthermore, HCWs and their family members are more likely to receive VIP treatment. For instance, when taken to the emergency department, children of physicians are less likely to have medical students and residents involved in their care and more likely to see attending physicians and consultants. ${ }^{8}$

In contrast, other categories of essential workers do not have such advantages. These workers are more likely to be members of marginalized racial and ethnic minority groups, have substantially lower wages, have less access to PPE, and work in more crowded conditions, and are less likely to have paid sick leave compared with $\mathrm{HCWs}^{7}{ }^{7}$ These workers are also more likely to lack access to quality healthcare. In fact, many safety net hospitals that provide care to marginalized communities have faced significant financial hardships as a result of the pandemic, and without additional support, some may close. Prioritizing HCWs will likely widen the gaps in health, economic, and social status among these groups.

With respect to allocation criteria, Black, Latinx, and Native American communities have more severe morbidity and mortality from COVID-19 as a result of racism and its interaction with other social determinants of health. Members of marginalized communities of color have a higher likelihood of becoming infected with COVID-19, a higher prevalence of comorbidities, and less access to treatment. ${ }^{7}$ Before her untimely death, Dr Susan Moore, a Black family physician, painfully described the racism to which she was subjected while being treated for COVID-19.9 The economic devastation caused by the pandemic, including unemployment, evictions, and food insecu- rity, compounds the impact of social determinants of health and disproportionately affects minority communities. Purely race- and ethnicity-based approaches to allocation to redress these inequities have potential limitations and obstacles, such as omission of other social determinants of health and legal challenges. ${ }^{7}$ While currently proposed for allocation of medications or vaccines, alternatives include using the Centers for Disease Control and Prevention's Social Vulnerability Index ${ }^{8}$ or the Area Deprivation Index ${ }^{10}$ as a priority criterion. Most importantly, healthcare systems should more broadly demonstrate themselves trustworthy and assure that marginalized communities of color have access to quality healthcare services.

\section{CONCLUSION}

The United States has failed to adequately control the COVID-19 pandemic, and increasing numbers of admissions and staffing shortages have renewed concerns that hospitals will need to implement crisis standards of care. Daffner ${ }^{4}$ argues that healthcare providers should be prioritized in the allocation of critical care based on reciprocity. In the current phase of the pandemic, HCWs are more likely to be infected by one another or in the community than by patients. There are also other ways that hospitals can discharge this duty that do not require prioritizing HCWs over patients. The category of HCW is both over- and underinclusive, and Daffner ${ }^{4}$ has not shown that prioritization can be implemented fairly. Finally, inordinate attention has been paid to this topic. Much more attention should be focused on how to redress the ways in which the pandemic has exacerbated existing racial and ethnic inequities.

Disclosures: The authors have nothing to disclose.

\section{References}

1. COVID data tracker: United States COVID-19 cases and deaths by state. Centers for Disease Control and Prevention. Updated February 22, 2021. Accessed February 22, 2021. https://covid.cdc.gov/covid-data-tracker/\#cases_casesper100klast7days

2. WHO coronavirus disease (COVID-19) dashboard. World Health Organization. Updated February 22, 2021. Accessed February 22, 2021. https://covid19.who.int/

3. Sins of omission: how government failures to track Covid-19 data have led to more than 1,700 health care worker deaths and jeopardize public health. National Nurses United. September 2020. Accessed February 11, 2021. https://act.nationalnursesunited.org/page/-/files/graphics/0920_Covid19_ SinsOfOmission_Data_Report.pdf

4. Daffner KR. Point: healthcare providers should receive treatment priority during a pandemic. J Hosp Med. 2021;16(3):180-181. https://doi. org/10.12788/jhm.3596

5. Wang D, Hu B, Hu C, et al. Clinical characteristics of 138 hospitalized patients with 2019 novel coronavirus-infected pneumonia in Wuhan, China. JAMA. 2020;323(11):1061-1069. https://doi.org/10.1001/jama.2020.1585

6. Seidelman JL, Lewis SS, Advani SD, et al. Universal masking is an effective strategy to flatten the severe acute respiratory coronavirus virus 2 (SARSCoV-2) healthcare worker epidemiologic curve. Infect Control Hosp Epidemiol. 2020:41(12):1466-1467. https://doi.org/10.1017/ice.2020.313

7. Gayle H, Foege W, Brown L, Kahn B, eds. Framework for Equitable Allocation of COVID-19 Vaccine. The National Academies Press; 2020. https://doi. org/10.17226/25917

8. Diekema DS, Cummings $P$, Quan L. Physicians' children are treated differently in the emergency department. Am J Emerg Med. 1996;14(1):6-9. https: //doi.org/10.1016/S0735-6757(96)90002-9

9. Maybank A, Jones CP, Blackstock U, Perry JC. Say her name: Dr. Susan Moore. The Washington Post. December 26, 2020. Accessed January 6, 2021. https: //www.washingtonpost.com/opinions/2020/12/26/say-her-name-dr-susan -moore/

10. White DB SchmidhoferM, McCreary E et al Model hospital policy for fair allocation of scarce medications to treat COVID-19. University of Pittsburgh. May 28, 2020. Accessed November 23, 2020. https://ccm.pitt.edu/sites/default /files/2020-05-28b\%20Model\%20hospital\%20policy\%20for\%20allocating \%20scarce\%20COVID\%20meds.pdf 